\title{
To discover the dust patterns from image in source camera identification-A survey
}

\author{
Manpreet Kaur $^{1}$, Reecha Sharma ${ }^{2}$ \\ ${ }^{1}$ M.tech student, Dept. Of ECE Punjabi University Patiala, India \\ ${ }^{2}$ Asst. Professor, Dept. of ECE Punjabi University Patiala, India
}

\begin{abstract}
The source camera identification is the technique in which we identify the problems that affect the images which are taken with digital cameras or mobile phones. The images which are taken with the professional cameras like DSLR are of high quality, but when the dust particles or other noise parameters introduced in the capturing device then it leads to create the dust patterns on the image which is captured. When the dust particles stuck on the captured image it reduces the image quality. In this paper, various techniques are discussed in which various features are detected from the captured image.
\end{abstract}

Keywords: Source camera identification; image forensics; PSNR; MSE.

\section{Introduction}

Image is a matrix in which elements are arranged in the form of rows and columns. Image Processing is a process of applying some operations on the image to get a modified image and obtaining the valid information from it. Image processing has many applications in the field of every business. There are so many problems which affect the image in terms of many parameters. To discuss we have to see first that how the image formed in the camera.

\subsection{Image formation in digital cameras}

As shown in the figure (see Fig 1.), firstly light enters to camera by the lens. Further filters are there. Then the block next to this is called the color filter array (CFA). Next to this section CCD array is arranged by using different filters, typically red, green and blue (RGB). CCD array is the most expensive component of a digital camera. Capturing the colour image process requires separate CCD arrays for each colour component. So this is the reason that $\mathrm{CCD}$ is expensive component of the digital camera.

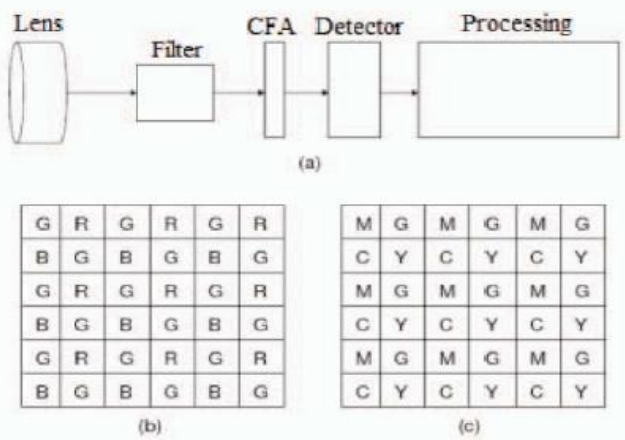

Fig 1: Image formation in cameras

Now one of the main problem which affect the quality of the image is dust patterns or we can say that a small noise. So following are some reasons which explains that how the dust or noise enters to the camera while capturing the image.

\subsection{Image with dust patterns}

When the lens of the camera is changed, dust enters to the camera body and settles on the image sensor. DSLRs are affected by this issue. Sometimes there are imperfections can be seen in the captured images like blurring, dead pixels or some important information is hidden due to the distorted image. So it is very important to analyse that problem so as to get the perfect image.

\subsubsection{Disadvantages of dust particles on image:}

Dust particles once land on the image sensor's surface they can affect the quality of all the images. There is also one main thing to know the difference between the dust pattern on the image and noise in the 
image. When pixel value difference is less from the original image then we can say that there are small dust patterns on the image and if the pixel difference is more, that leads to the noise present in the image.

\section{Review Process}

In [1], a patch-based (PB) SPN is extracted from the image and then algorithm is implemented.

Authors proposed a method based upon the detection of the dust particles [2]. Dust spots in the image are detected based on shape properties.

In [3], method proposed by averaging the noise obtained from multiple noisy images. Then a reference pattern noise is taken out as a watermark, which is considered by using a correlation detector.

In this study many techniques are divided for the identification: Metadata, CFA and Demosaicing Artifacts, Image Features. The main idea is to describe each category in detail. [4]

In this paper the approach to find the source camera depends upon the image features and the classification using SVM. [5]. The images used for the detection are clicked mainly by the different mobile devices.

In [6], the survey focused to interpolate the image to figure out the image characteristic values with SVM classifier and to obtain high detection rate.

Authors proposed a new camera identification method based upon the sensor dust characteristics. Dust particles on the image are detected using shape features to form the dust pattern on the DSLR camera. [7].

In [8], authors identified the source camera of an image based on the proprietary interpolation algorithm. A set of image characteristics are defined and then used with support vector machine.

III. Comparison Table

Table1. Comparison of papers

\begin{tabular}{|c|c|c|c|c|c|c|}
\hline $\begin{array}{l}\text { Papa name } \\
\text { (see reference) }\end{array}$ & $\begin{array}{l}\text { Year of } \\
\text { publication }\end{array}$ & $\begin{array}{l}\text { Method \& } \\
\text { experiment }\end{array}$ & $\begin{array}{l}\text { Image size } \\
\text { used }\end{array}$ & $\begin{array}{l}\text { Features } \\
\text { considered }\end{array}$ & Accuracy & Future scope \\
\hline$[8]$ & 2005 & $\begin{array}{l}\text { Proprietary interpolation } \\
\text { algorithm } \\
\text { EM algorithm } \\
\text { (expectation/ } \\
\text { maximization) }\end{array}$ & $1600 \times 1200$ & Auto-focus & $89.3 \%$ & $\begin{array}{l}\text { To solve the } \\
\text { images that are } \\
\text { heavily } \\
\text { compressed }\end{array}$ \\
\hline [3] & 2006 & $\begin{array}{ll}\text { Extracting } & \text { reference } \\
\text { pattern noise } & \end{array}$ & $1200 \times 1792$ & ----- & ----- & $\begin{array}{l}\text { Identification of } \\
\text { video cameras and } \\
\text { scanners }\end{array}$ \\
\hline [2] & 2008 & $\begin{array}{l}\text { Scheme based on sensor } \\
\text { dust traces. }\end{array}$ & $800 \times 533$ & High focal number & $92 \%$ & $\begin{array}{l}\text { Detection of dust } \\
\text { spots in very } \\
\text { complex regions } \\
\text { and low f- } \\
\text { numbers. }\end{array}$ \\
\hline [7] & 2009 & $\begin{array}{l}\text { Support vector machine } \\
\text { classifier }\end{array}$ & $\begin{array}{l}1024 \times 1024 \\
512 \times 512\end{array}$ & $\begin{array}{lr}\text { Sharpness r and } \\
\text { ISO(image } & \text { sensor } \\
\text { optimization)sensiti } \\
\text { vity }\end{array}$ & ---- & ---- \\
\hline [6] & 2012 & $\begin{array}{l}\text { Figure out the image } \\
\text { characteristic value }\end{array}$ & ---- & ---- & $90 \%$ & ----- \\
\hline [4] & 2013 & $\begin{array}{l}\text { Demosaicing Artifacts, } \\
\text { Lens Distortions and } \\
\text { Wavelet Transforms }\end{array}$ & ----- & ----- & ----- & $\begin{array}{l}\text { To bridge the } \\
\text { remaining gaps. }\end{array}$ \\
\hline [5] & 2015 & $\begin{array}{l}\text { Image features and } \\
\text { classification using } \\
\text { support vector machines }\end{array}$ & $1024 \times 768$ & Mobile devices & $80.69 \%$ & $\begin{array}{l}\text { To optimize the } \\
\text { success rate for } \\
\text { different cases } \\
\text { presented with } \\
\text { mobile device } \\
\text { images }\end{array}$ \\
\hline [1] & 2015 & $\begin{array}{lr}\text { a novel patch-based } \\
\text { (PB) sensor pattern } \\
\text { noise algorithm for } \\
\text { camera } & \text { source } \\
\text { identification } & \text { is } \\
\text { proposed } & \end{array}$ & $\begin{array}{l}256 \times 256 \\
512 \times 512\end{array}$ & $\begin{array}{l}\text { Smooth image } \\
\text { regions }\end{array}$ & $70-80 \%$ & \\
\hline
\end{tabular}

\subsection{Dust detection}

\section{Existing Method Result}

In the existing method an approach is based on different types of image features. We have also tried to detect the dust patterns by taking out the RGB patterns. We have also calculated the PSNR and MSE values of the dusty image. Accuracy can also be seen after this experiment. 


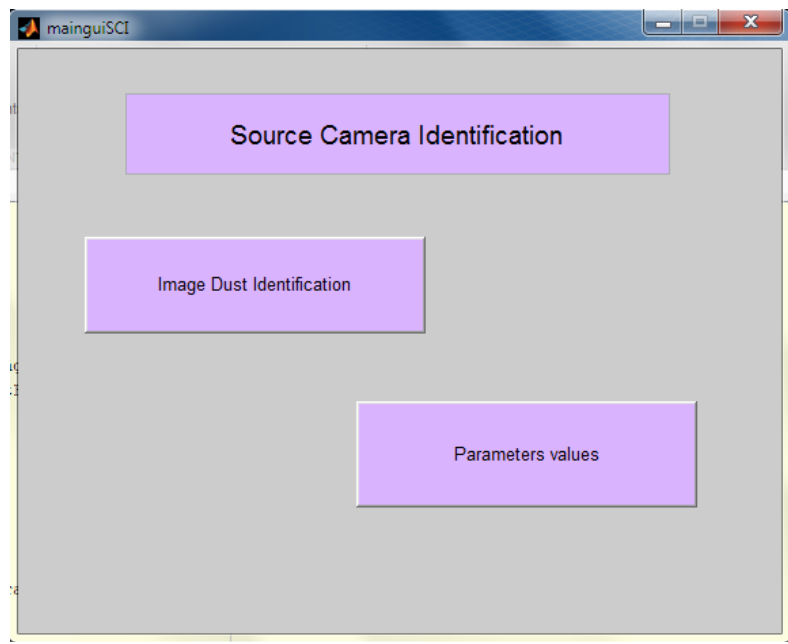

Fig 2: user interface setup

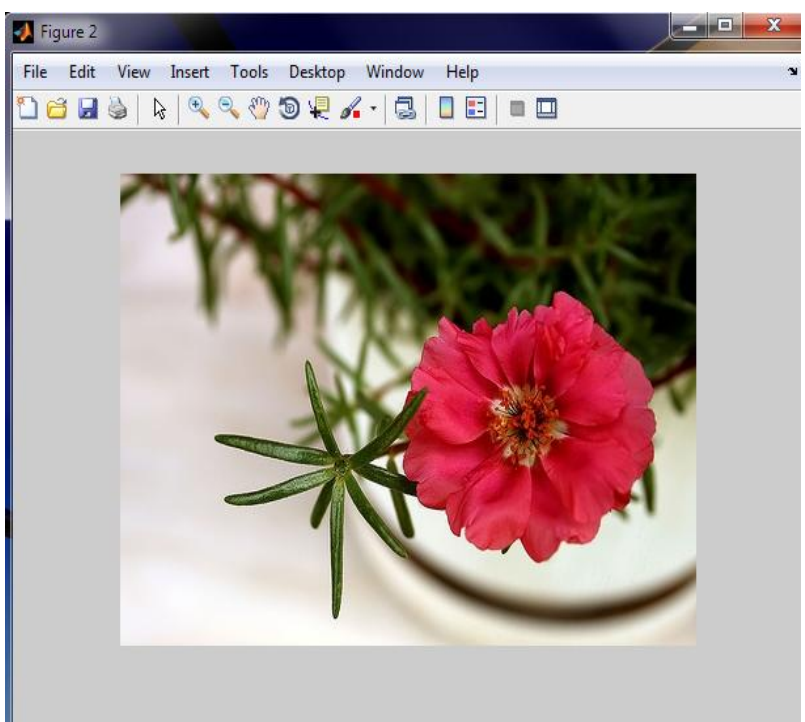

Fig 3: original image

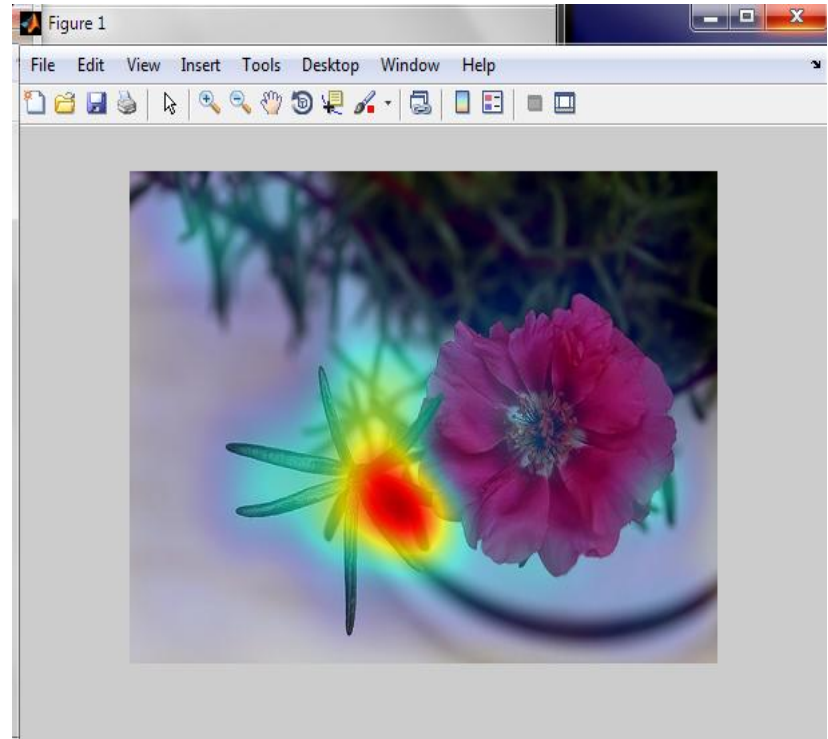

Fig 4: dust detected image 


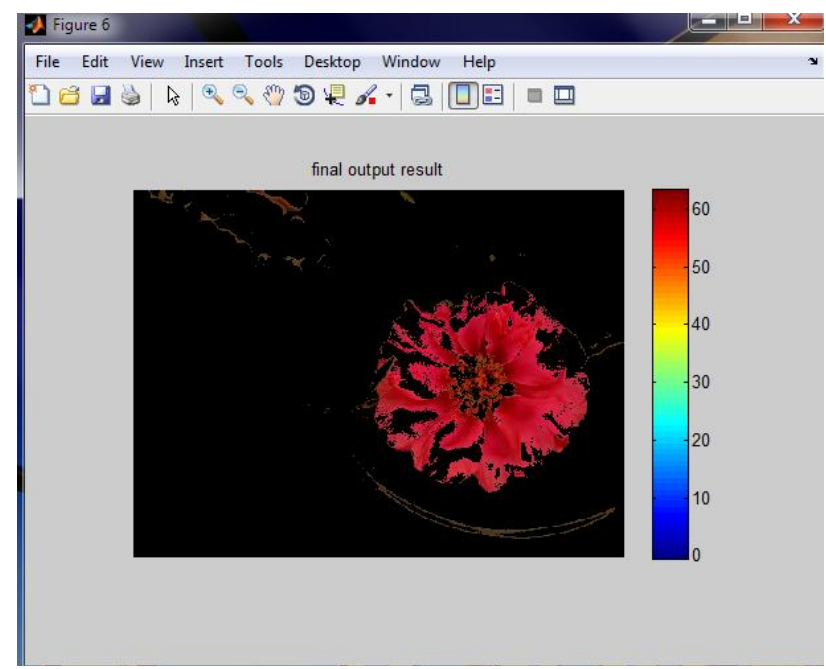

Fig 5: dust detected on red pattern only

Table 1.Parameter values

\begin{tabular}{|l|l|}
\hline Parameters & Values \\
\hline PSNR & 17.53851 \\
\hline MSE & 1155.12498 \\
\hline Fault rate Dust Detection & 0.15956 \\
\hline Accuracy & 82.97178 \\
\hline
\end{tabular}

\section{Future Work}

Future work will be focused on to detect dust patterns from the image on the B(blue) and G(green) area out of the RGB pattern which is not defined in the present work. Also to increase the accuracy of detecting the dust patterns.

\section{Conclusion}

When the image is captured by the camera, dust patterns added to the image and this leads in reduction of the image quality. In this paper, various techniques are discussed regarding source camera identification and regarding the issues related to the quality of an image. In the proposed technique whole image scanned which will leads to generation of dust patterns on the B \& G part out of the RGB pattern image. This technique also leads to improve image quality in terms of accuracy and moreover in MSE and PSNR.

\section{References}

[1]. Yue Tan, Bo Wang, Meijuan Zhao, Xiangwei Kong, and Ming Li, Patch-based sensor pattern noise for camera source identification. School of Information and Communication Engineering, Dalian University of Technology, Dalian, Liaoning, 116024, P. R. China.

[2]. Ahmet Emir Dirik, Husrev Taha Sencar, and Nasir Memon, "Digital single lens reflex camera identification from traces of sensor dust" IEEE Transactions on Information Security and Forensics and security, vol. 3, no. 3, Sep 2008.

[3]. J. Lukac, J. Fridrich, and M. Goljan, "Digital camera identification from sensor pattern noise," IEEE Transactions on Information Security and Forensics, vol. 1, pp. 205-214, 2006.

[4]. Ana Lucila Sandoval Orozco ${ }^{1}$, Jocelin Rosales Corripio ${ }^{1}$, David Manuel Arenas González, Luis Villalba ${ }^{1}$ Hernández Castro $^{2}$, "Techniques for source camera identification.

[5]. Ana Lucila Sandoval Orozco ${ }^{1}$, Jocelin Rosales Corripio ${ }^{1}$, David Manuel Arenas González, Luis Villalba ${ }^{1}$ Hernández Castro $^{2}$, "Image source acquisition identification of mobile devices based on the use of features", Springer Science+Business Media New York 2015.

[6]. Cheng-Liang Lai, Ching-Yi Lin, "Source Camera of Digital Image Identification based on Interpolation Algorithm", Department of Applied Informatics, Fo Guang University

[7]. No.160, 2012.

[8]. Yanmei fang, A.E. dirik, Xiaoxi sun, Nasir memon, "Source class identification for DSLR and compact cameras", IEEE'2009

[9]. Sevinc Bayram a, Husrev T. Sencar b, NasirMemon b, Ismail Avcibas, "source camera identification based on cfa interpolation". IEEE 2005. 